\title{
An imprinted signature helps isolate ESC-equivalent iPSCs
}

\author{
Ernesto Lujan $^{1,2}$, Marius Wernig ${ }^{1}$ \\ ${ }^{I}$ Institute for Stem Cell Biology and Regenerative Medicine, Department of Pathology; ${ }^{2}$ Department of Genetics, Stanford \\ University School of Medicine, 1050 Arastradero Road, Palo Alto, California 94304, USA \\ Cell Research (2010) 20:974-976. doi:10.1038/cr.2010.117; published online 10 August 2010
}

Since the demonstration of direct reprogramming of differentiated cells such as fibroblasts, to a malleable, pluripotent state by defined transcription factors great effort has been given to isolate induced pluripotent stem cells (iPSCs) with the same developmental potential as embryonic stem cells (ESCs) derived from blastocysts. Various selection and morphological criteria have led to the isolation of iPSCs with differential pluripotent capacity, but without the addition of small molecules very few lines have been able to undergo the most stringent pluripotent test - generate viable "all iPS cell mice" by tetraploid complementation. In a recent elegant study, Stadtfeld and colleagues have proposed that silencing of the imprinted Dlk1-Dio3 gene cluster is responsible for this variability of pluripotency potential in iPSCs, and the key to isolating ESC equivalent iPSCs [1].

Demonstration of pluripotency largely rests on four criteria (in order of increasing stringency): 1) teratoma formation following subcutaneous injection and demonstration of the presence of tissues representing all three germ layers; 2) ability to contribute to chimeric animals following blastocyst injection; 3) germline transmission; and 4) "all iPS cell mice", i.e. full embryo contribution by tetraploid $(4 \mathrm{~N})$

Correspondence: Marius Wernig

E-mail: wernig@stanford.edu embryo complementation. The latter assay involves the injection of diploid cells into $4 \mathrm{~N}$ blastocysts so that only the injected cells can contribute to the developing embryo properly whereas the $4 \mathrm{~N}$ host cells are believed to solely contribute to extraembryonic placental tissue. The first generation iPSC lines were generated through introduction of the four reprogramming factors Oct4, Sox 2, Klf4, and $c-M y c$ and selection for the reactivation of the ES cell-specific gene Fbx15 [2]. These cells resembled ES cells morphologically and formed teratomas, but could not contribute to adult chimeric mice. Furthermore, many of the clones failed to show demethylation and reactivation of the key pluripotency genes Oct4 and Nanog suggesting a lack of reprogramming at an epigenetic level. Therefore the optimization of reprogramming methods to yield iPSCs that are fully equivalent to ES cells has been the subject of intense investigation since.

Focusing on isolating ESC-equivalent iPSCs, and in an effort to cull away some heterogeneity, three independent groups derived second generation iPSCs through selection of Oct4 and Nanog reactivation [3-5]. Both genes are necessary for pluripotency maintenance, in contrast to the Oct4 downstream target gene Fbx15. Most clones derived from these lines exhibited demethylation of the Oct4 and Nanog promoters, could form teratomas, and, importantly, were able to contribute to live high-grade chimeras that survived to adulthood. Moreover some lines also formed "all-iPS cell embryos" by $4 \mathrm{~N}$ embryo complementation surviving until day 14.5 of development [3]. While these iPSC lines showed "better" pluripotent potential than the $F b x 15$ selected lines, none of the embryos derived via $4 \mathrm{~N}$ complementation survived to adulthood, even in the 129/C57B6 F1 genetic background that is known to be permissive for $4 \mathrm{~N}$ complementation. Thus, second generation iPSCs were almost but not completely identical to ES cells. At this point, it should be noted that ES cells generated by nuclear transfer-mediated reprogramming (NT-ES cells) from adult somatic cells are indistinguishable from normal ES cells including the parameter of $4 \mathrm{~N}$ complementation. Surprisingly, last year, several groups succeeded to derive iPSC lines that could generate live pups via $4 \mathrm{~N}$ complementation at levels comparable to ESCs [6-8], thus demonstrating that indeed, ESC equivalent pluripotent cells could be generated from differentiated cells with the induction of one to four reprogramming factors. Unfortunately, without chemical inhibitors, these lines seemed to be rare - even with stringent Oct4 selection and morphological criteria, more than $70 \%$ of iPSC lines isolated and tested did not have the capacity to generate a live adult by $4 \mathrm{~N}$ complementation [6]. Thus, different iPS cell clones seem very heterogeneous. Compounded with 
a recent study that found consistent differences in expression levels for $\sim 300$ genes in iPSCs when compared to ESCs [9], the question remained whether there was a fundamental difference between these ESC equivalent iPSCs. Building on this platform of heterogeneity, Stadtfeld and colleagues surprisingly isolated a single locus that correlates with iPSC developmental potential, and may provide the key to isolating ESCequivalent iPSCs.

Efforts to identify differences by expression profiling and meta-analysis have found signature dissimilarities between ESCs and iPSCs, they have failed to find a key difference to separate the two populations [9]. Reasoning that background noise from ESCs and iPSCs derived from genetically distinct animals used in these analyses might be the reason for this lack of coherence, Stadtfeld and colleagues sought to compare populations with the same genetic background. The authors compared iPSCs that were derived from a mouse that contained the four reprogramming factors under doxycycline control to ES cells that were used to generate those mice [10]. Thus, these iPSCs were clonally derived from their respective ES cells and genetically identical. Surprisingly, a comparison of expression and microRNA profiles between the two populations showed that expression levels for only two genes, Gtl2 and Rian, and 21 miRNAs differed significantly- a small number compared to the $\sim 300$ genes previously reported to be differentially expressed between ESCs and iPSCs [9]. These genes and microRNAs were downregulated in iPSCs and surprisingly localized to a single genomic locus, the Dlk1-Dio3 imprinted gene cluster.

To rule out that repression at the Dlk1-Dio3 gene cluster was unique to iPSCs derived from embryonic fibroblasts, Gtl2 expression levels were assessed by qPCR and found to be equally negligible in iPSCs originating from hematopoietic stem cells, granulocyte- macrophage progenitors, granulocytes, and keratinocytes. Alongside these tissues, Gtl2 levels were also evaluated in iPSCs generated from perinatal and adult fibroblasts, and surprisingly while most iPSCs generated from these tissues also showed equally low levels of Gtl2 expression, several of these iPSCs had Gtl2 transcript levels comparable to those of ESCs, providing evidence for two subtypes of iPSCs - Gtl $2^{\mathrm{on}}$ and Gtl2 ${ }^{\text {off }}$. Further analysis of this region showed signatures of complete epigenetic silencing in $G t 2^{\text {off }}$ iPSCs as shown by an increase in DNA methylation in the region unlike ESCs and the donor fibroblasts. Functional assessment of the two iPSC populations by injection into $2 \mathrm{~N}$ and $4 \mathrm{~N}$ blastocysts showed that while $G t 2^{\text {off }}$ iPSCs contributed to low grade chimeras and failed to generate embryos to term via $4 \mathrm{~N}$ complementation, Gtl ${ }^{\circ \mathrm{on}}$ iPSCs contributed to high grade chimeras and generated live pups at efficiencies similar to ESCs via $4 \mathrm{~N}$ complementation. With this, Statdfeld and colleagues seemed to have isolated a key region that correlated with ESCequivalent iPSCs.

So what is this Dlk1-Dio3 gene cluster? Located on chromosome 12qF1, the Dlkl-Dio3 gene cluster is associated with a differentially methylated region (DMR) that is normally imprinted paternally. The methylation status of this DMR acts as an imprinting control region of the entire cluster. This is particularly interesting given that it has been thought that imprinting, a process where maternal or paternal genes are selectively silenced, only occurs in the germline in mammals and other imprinted regions were not changed by direct reprogramming [3]. Supporting this notion, expression data from the current study suggests that silencing at imprinted loci in $4 \mathrm{~N}$ incompetent $\mathrm{PPSCs}$ is not a general phenomenon, but is selective to the Dlk1-Dio3 region. Since nuclear transfer mediated reprogramming with somatic cells consistently yields cells that are $4 \mathrm{~N}$ competent, an attempt was made to rescue this phenotype by nuclear transfer with $\mathrm{Gtl}^{\text {off }}$ donor iPSC nuclei. As expected, this attempt was unsuccessful because imprinted marks are typically not affected following nuclear transfer. Thus the silencing at the Dlk-Dio3 gene cluster is a unique product of reprogramming using the "Yamanaka method".

Though Stadtfeld and colleagues make a clear case for a correlation between ESC-equivalent iPSCs and a lack of silencing at the Dlk1-Dio3 gene cluster, it is still not fully conclusive whether this one single faulty epigenetic mark causes iPSCs to be incompetent. While only genes and miRNAs localized to the Dlk1-Dio3 gene cluster were repressed in $4 \mathrm{~N}$ incompetent iPSCs, it remains to be addressed whether anything silenced in this region can actually rescue this phenotype or if silencing at this locus is just a proxy of noncanonical genomewide repression at selective loci whose expression levels would not be detected in iPSCs but later in development; it should be noted that most embryos derived from $4 \mathrm{~N}$ incompetent $i \mathrm{PSCs}$ arrest late in development, suggesting that it is a lack of developmental potential - and most likely transcripts expressed during this later stages - rather than a defect in an early pluripotent state that leads to arrest. Methylation and acetylation maps of genetically matched ESCs and iPSCs would be a valuable tool in this endeavor. Additionally, while expression of Gtl2 could be rescued by treatment with a histone deacetylase, valproic acid (VPA), in Gtl2 ${ }^{\text {off }}$ iPSCs, viable mice could not be made from these iPSCs, possibly due to other effects of VPA; interestingly another group has reported that $100 \%$ of iPSCs derived from embryonic fibroblasts with continuous VPA treatment during the first 23 days of reprogramming could generate viable adult mice by $4 \mathrm{~N}$ complementation [8]. To address the specificity of this defect, a defined genetic or epigenetic manipulation of the Dlk1-Dio3 locus needs to be performed. It might be informative 
whether the artificial silencing of that locus renders ES cells incompatible with $4 \mathrm{~N}$ embryo complementation. Further refinement of the cause of iPSC competency may lead to better diagnostic measures that will help isolate ESCequivalent iPSCs from what is currently a heterogeneous pool.

Stadtfeld and colleagues have provided an incredibly interesting lead to isolating ESC-equivalent iPSCs from a heterogeneous population. That the key difference between these two populations is silencing at an imprinted locus is very surprising and unexpected. All reprogramming events - including the one during early embryogenesis - are considered to be genome wide but spare the paternal and maternal imprints. The erasure and imprinting of these loci is unique to the germ line. Further research will be needed to understand the mechanism for this and how the Yamanaka factors lead to a change of an imprint that must involve molecular machinery specific for the germ line. The possibility of silencing at an imprinted locus during reprogramming also leaves many open questions for the field such as line specificity, locus specificity, timing, and whether this occurs in humans. An expansion of these findings in other contexts will give a clearer picture of the impact of this phenomenon during reprogramming, but even without these answers, we are one substantial step closer to isolating ESC-equivalent iPSCs with ease.

\section{References}

1 Stadtfeld M, Apostolou E, Akutsu H, et al. Aberrant silencing of imprinted genes on chromosome $12 \mathrm{qF} 1$ in mouse induced pluripotent stem cells. Nature 2010; 465:175-181.

2 Takahashi K, Yamanaka S. Induction of pluripotent stem cells from mouse embryonic and adult fibroblast cultures by defined factors. Cell 2006; 126:663676.

3 Wernig M, Meissner A, Foreman R, et al. In vitro reprogramming of fibroblasts into a pluripotent ES-cell-like state. Nature 2007; 448:318-324.

4 Okita K, Ichisaka T, Yamanaka S. Gen- eration of germline-competent induced pluripotent stem cells. Nature 2007; 448:313-317.

5 Maherali N, Sridharan R, Xie W, et al. Directly reprogrammed fibroblasts show global epigenetic remodeling and widespread tissue contribution. Cell Stem Cell 2007; 1:55-70

6 Zhou XY, Li W, Lv Z, et al. iPS cells produce viable mice through tetraploid complementation. Nature 2009; 461:86-90.

7 Kang L, Wang J, Zhang Y, Kou Z, Gao S. iPS cells can support full-term development of tetraploid blastocystcomplemented embryos. Cell Stem Cell 2009; 5:135-138.

8 Boland MJ, Hazen JL, Nazor KL, et al. Adult mice generated from induced pluripotent stem cells. Nature 2009; 461:91-94.

9 Chin MH, Mason MJ, Xie W, et al. Induced pluripotent stem cells and embryonic stem cells are distinguished by gene expression signatures. Cell Stem Cell 2009; 5:111-123.

10 Stadtfeld M, Maherali N, Borkent M, Hochedlinger K. A reprogrammable mouse strain from gene-targeted embryonic stem cells. Nat Methods 2010; 7:53-55. 